Jakub Źrebiec*

\title{
Modelling of Unmanned Aerial Vehicle - Tricopter
}

\section{Introduction}

Nowadays, the interest of Unmanned Aerial Vehicles (UAV) is ameliorating very quickly, both in civilian and military usage. The UAVs have great potential in areas such as: agriculture, geodesy, inspection, energetics and entertainment. The most popular type of drone is quadrocopter both in scientific and commercial aspects. Quadorotor has four engines with propellers and occurs in variety sizes and weights. From very small, which can be placed on hand, up to $1.5-2 \mathrm{~m}$ in diameter and they are able of carry payloads from few grams to even $20 \mathrm{~kg}$.

This paper describes modelling Tricopter - uav with three rotors. Trirotors, rather then quadrocopters, are less popular, due to system instability - autorotataion in yaw axis. This effect occurs because aerodynamic moments are unbalanced. It can be eliminated by change in construction, one of motors must be tilted. The best approach is have possibility to change angle of tilted motor, during flight.

Tricopter and other multirotors are capable of vertical taking-off and landing (VTOL), hovering flight. But, in contrary of multicopters with even rotors number, Trirotors have greater flight dynamics and improved manoeuvrability (due to built-in instability).

This study presents, more detailed, mathematical models of Tricopter's subsystems: BLDC (brushless direct current) motor with propeller and Li-poly battery. Moreover, also presented a flight dynamics model which is based on quaternions algebra. However, this model has lack of aerodynamics coefficients, so it can be used only for stability analysis in hovering flight.

* AGH University of Science and Technology, Department of Automatics and Biomedical Engineering, Krakow, Poland 


\section{Propeller, aerodynamics forces and moments}

Consider propeller of diameter $D$, which has angular speed $\omega_{s}$ (Fig. 1). This movement generate thrust $\vec{T}$ directed perpendicular to the plane of rotation, torque $\vec{M}_{S}$ acting on the motor's shaft, and torque $\vec{M}_{P}$ effecting on Tricopter's yaw axis. To calculate thrust $\vec{T}$ and torque $\vec{Q}$ blade element theory is used. It says that propeller's blade consists of infinitely many, infinitely thin and aerodynamically independent elements, which are located along the radius. On Figure 1 sample section is marked. It has thickness $\mathrm{dr}$ and is placed in $\mathrm{r}$ from centre, whereas Figure 2 shows cross section of the blade. The following forces have been marked on it: drag $-d D$, lift $-d L$, thrust $-d T$ and torque $-d Q$. Also marked: forward speed $-v$, angle of attack $-\alpha$ and effective pitch angle $-\xi$. There is more parameters, such as inducted speed or pitch angle, all of them can be found in [2].

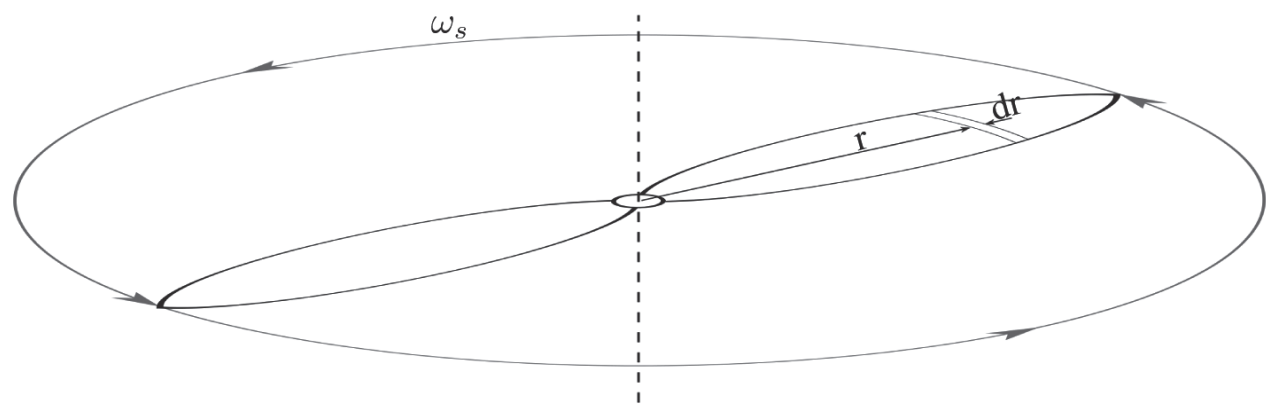

Fig. 1. Propeller

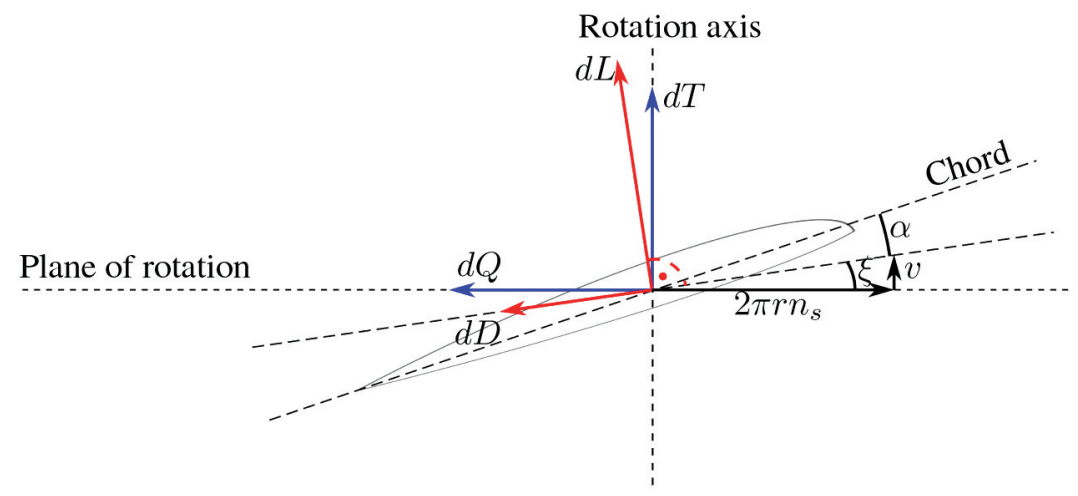

Fig. 2. Cross section of the blade 
Based on Figure 2, forces $d T$ and $d Q$ can be expressed using $d L$ and $d D$,

$$
\begin{aligned}
& d T=d L \cos \xi-d D \sin \xi \\
& d Q=d L \sin \xi+d D \cos \xi
\end{aligned}
$$

while forces $\mathrm{dL}$ and $\mathrm{dD}$ have the following form $[2,6,11]$ :

$$
\begin{aligned}
& d L=\frac{1}{2} \rho v_{R}^{2} l c_{L} d r \\
& d D=\frac{1}{2} \rho v_{R}^{2} l c_{D} d r
\end{aligned}
$$

where:

$\rho$ - air density,

$l$ - average (for simplified) chord's length,

$c_{L}, c_{D}-$ lift and drag coefficients.

To calculate thrust $T$ and torque $M_{S}$, perform following actions:

$$
\begin{aligned}
& T=N_{B} \int_{0}^{R} d T d r \\
& M_{S}=N_{B} \int_{0}^{R} r d Q d r
\end{aligned}
$$

where $N_{B}$ is number of blades. Way of solving integrals from equations (5) and (6) can be found in $[2,6,11]$. The result is:

$$
\begin{aligned}
& T=C_{T} \rho n_{s}^{2} D^{4} \\
& M_{S}=C_{M} \rho n_{S}^{2} D^{5}
\end{aligned}
$$

where $C_{T}$ and $C_{M}$ is dimensionless coefficients of thrust and torque, $\rho$ is air density, $n_{S}$ is revolutions per second and $D$ is propeller's diameter. Manufactures provides coefficient $C_{P}$ instead $C_{M}$, which is dimensionless coefficient of power. Equation (11) presents relationship between them.

$$
P=M_{S} \omega_{S}=M_{S} 2 \pi n_{S}=2 \pi C_{M} \rho n_{S}^{3} D^{5}
$$




$$
\begin{aligned}
& P=C_{P} \rho n_{s}^{3} D^{5} \\
& C_{P}=2 \pi C_{M}
\end{aligned}
$$

Coefficients $C_{T}$ and $C_{P}$ are dependent on the coefficient - advance ratio from equation (12):

$$
J=\frac{v}{n_{s} D}
$$

Figure 3 shows relationship of coefficients $C_{T}$ and $C_{P}$ from $J$.

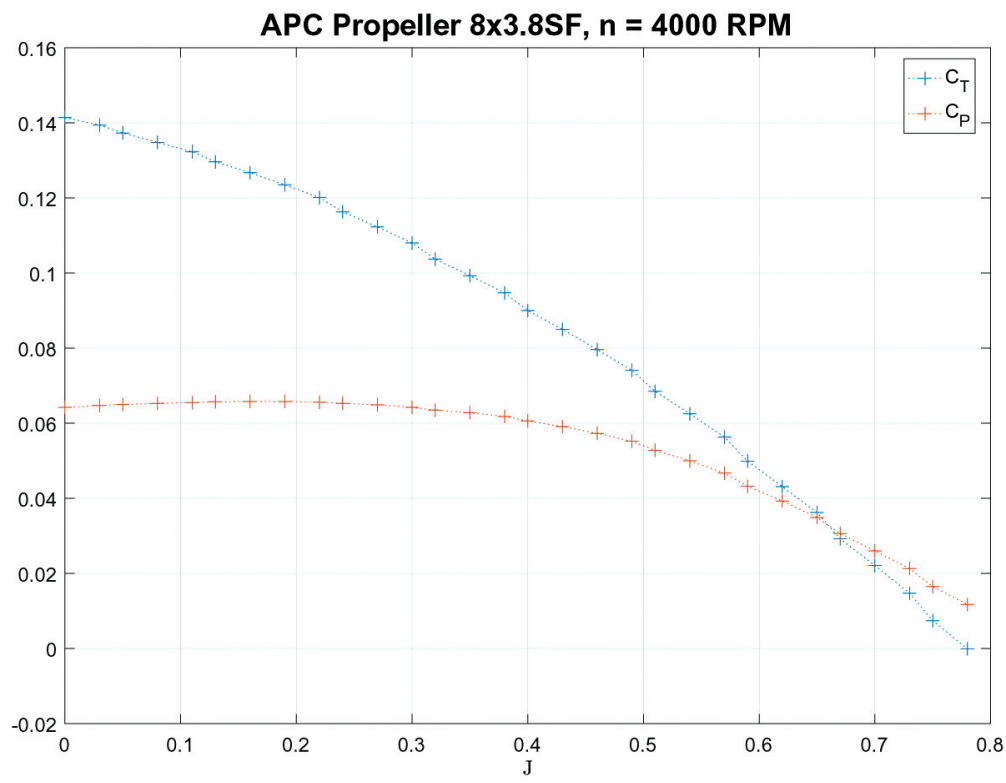

Fig. 3. Typical relationship of coefficients $C_{T}$ and $C_{P}$ from $J$

Torque $M_{P}$ acting on Tricopter's yaw axis is produced by force $d Q$ which is caused by propeller's rotation with speed $\omega_{s}$. Given that the propeller has two blades, so torque $M_{P}$ is sum of two torques. On the Figure 4 shows one of Tricopter's drive units which is place in distance $d$ from centre of mass. Based on Figure 4, torque produced by upper blade is equal to:

$$
\begin{aligned}
M_{P G} & =d Q \sin \left(\pi-\frac{\pi}{2}-\beta\right) \sqrt{r^{2}+d^{2}-2 r d \cos \varphi}= \\
& =d Q \cos \beta \sqrt{r^{2}+d^{2}-2 r d \cos \varphi}
\end{aligned}
$$




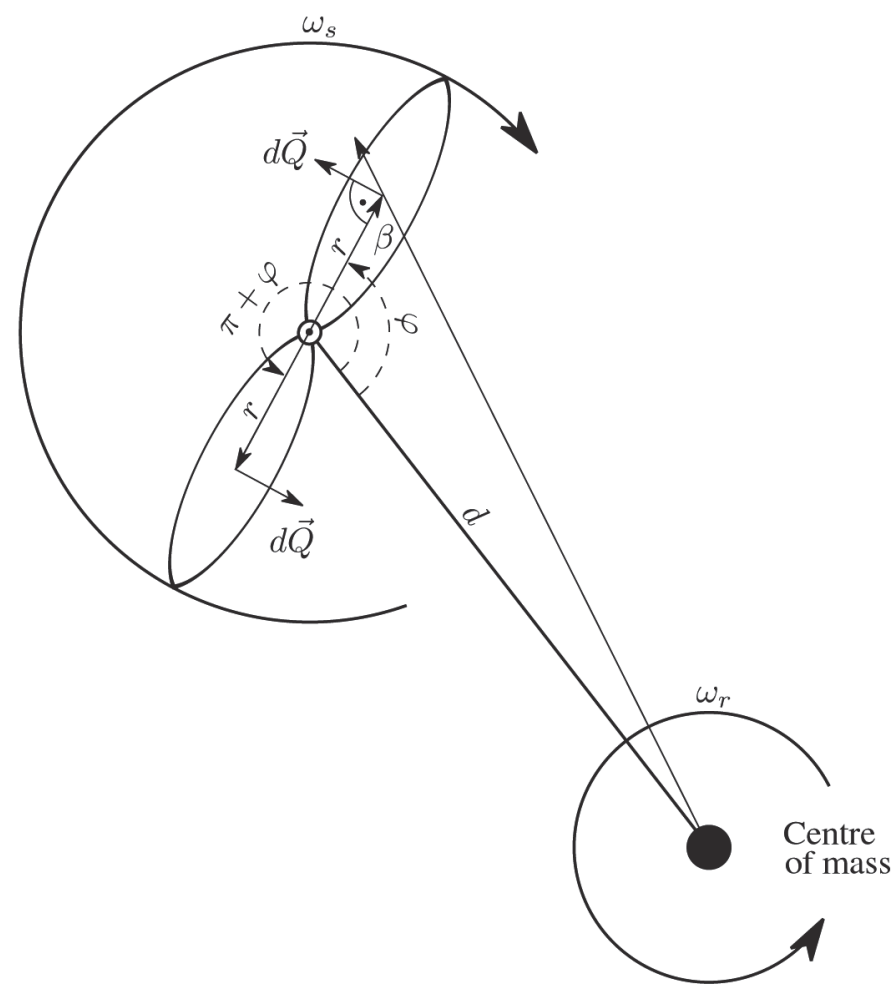

Fig. 4. One of Tricopter's drive units

And for the lower blade:

$$
\begin{aligned}
M_{P D} & =d Q \sin \left(\frac{\pi}{2}-\beta^{\prime}\right) \sqrt{r^{2}+d^{2}-2 r d \cos (2 \pi-\pi-\varphi)}= \\
& =d Q \cos \beta^{\prime} \sqrt{r^{2}+d^{2}+2 r d \cos \varphi}
\end{aligned}
$$

In next step, $\cos \beta$ i $\cos \beta^{\prime}$ must be calculated. From law of cosines:

$$
d^{2}=r^{2}+r^{2}+d^{2}-2 r d \cos \varphi-2 r \sqrt{r^{2}+d^{2}-2 r d \cos \varphi} \cos \beta
$$

which for $r>0$ gives:

$$
\cos \beta=\frac{r-d \cos \varphi}{\sqrt{r^{2}+d^{2}-2 r d \cos \varphi}}
$$


And analogously:

$$
\cos \beta^{\prime}=\frac{r+d \cos \varphi}{\sqrt{r^{2}+d^{2}+2 r d \cos \varphi}}
$$

After inserting equations (16) and (17) into (13) and (14):

$$
\begin{aligned}
& M_{P G}=d Q(r-d \cos \varphi) \\
& M_{P D}=d Q(r+d \cos \varphi)
\end{aligned}
$$

And finally obtained equation:

$$
\begin{aligned}
M_{P} & =2 \int_{\frac{\pi}{2}}^{\frac{3 \pi}{2}} \int_{0}^{R}\left(M_{P G}-M_{P D}\right) d r d \varphi= \\
& =-4 d \int_{\frac{\pi}{2}}^{\frac{3 \pi}{2}} \int_{0}^{R} d Q \cos \varphi d r d \varphi
\end{aligned}
$$

Way to solve the equation (20) can be found in [20]. The result is as follows:

$$
M_{P}=C_{M_{P}} d \rho n_{s}^{2} D^{4}
$$

where $C_{M_{P}}$ is dimensionless coefficient of torque acting on Tricopter's yaw axis, and which is dependent on $J$.

\subsection{BLDC motor}

In almost all copters available on the market, brushless direct current motors are used. They are the most important system on board, because they are the only source of thrust. BLDC motors can be divided into two types outrunners and inrunners. First type has magnets on outer, spinning shell and coils inside, while inrunners have inverse construction. BLDC motors needs three phase inverter to run. On Figure 5 has been shown equivalent circuit of motor. 


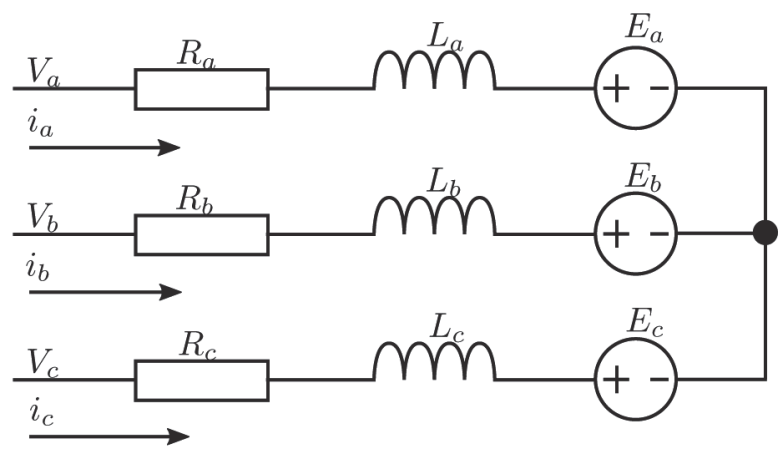

Fig. 5. Equivalent circuit of BLDC motor

There are a lot of literature about modelling brushless motors and control strategies for example $[4,8,10,12,15]$. Based on cited literature and Figure 5, the following equations were written:

$$
\begin{aligned}
& V_{a b}=R\left(i_{a}-i_{b}\right)+L \frac{d}{d t}\left(i_{a}-i_{b}\right)+E_{a}-E_{b} \\
& V_{b c}=R\left(i_{b}-i_{c}\right)+L \frac{d}{d t}\left(i_{b}-i_{c}\right)+E_{b}-E_{c} \\
& V_{c a}=R\left(i_{c}-i_{a}\right)+L \frac{d}{d t}\left(i_{c}-i_{a}\right)+E_{c}-E_{a} \\
& M_{e}=\mathbb{I}_{s} \frac{d \omega_{s}}{d t}+B \omega_{s}+M_{L}
\end{aligned}
$$

where:

$V$ - phase-to-phase voltage,

$i$ - phase current,

$E$ - phase electromotive force.

Moreover $R$ means phase-to-phase resistance $R=R_{a}+R_{b}=R_{b}+R_{c}==R_{c}+R_{a}$ and $L$ is phase-to-phase inductance $L=L_{a}+L_{b}=L_{b}+L_{c}=L_{c}++L_{a}$. $M_{e}$ is torque, $I_{s}$ is inertia of motor, $\omega_{s}$ is angular speed, $B$ is damping coefficient and $M_{L}=\mathbb{I}_{P} \frac{d \omega_{S}}{d t}+M_{S}$ is torque produced by load, in which $\mathbb{I}_{P}$ is inertia of propeller, $M_{S}$ is aerodynamic torque. 
Electromotive force of brushless, three phase direct current motor is function of rotor position, furthermore is shifted in phase $120^{\circ}$ for each phase $[8,10]$.

$$
\begin{aligned}
& E_{a}=k_{e} \omega_{s} f\left(\theta_{e}\right) \\
& E_{b}=k_{e} \omega_{s} f\left(\theta_{e}-\frac{2 \pi}{3}\right) \\
& E_{c}=k_{e} \omega_{s} f\left(\theta_{e}+\frac{2 \pi}{3}\right)
\end{aligned}
$$

where $\theta_{e}$ is electrical angle, $k_{e}$ is electrical constant $\left[\frac{V_{S}}{\mathrm{rad}}\right], \omega_{s}$ is angular speed and function $f\left(\theta_{e}\right)$ describes trapezoidal form.

Additionally electrical torque may be expressed as $[8,10]$ :

$$
M_{e}=k_{i}\left[f\left(\theta_{e}\right) i_{a}+f\left(\theta_{e}-\frac{2 \pi}{3}\right) i_{b}+f\left(\theta_{e}+\frac{2 \pi}{3}\right) i_{c}\right]
$$

where $k_{i}$ is torque constant $\frac{\mathrm{Nm}}{\mathrm{A}}$.

Relationship between electrical angle and angular speed is expressed as follows:

$$
\begin{gathered}
\frac{d \theta_{e}}{d t}=\frac{P}{2} \omega_{s} \\
\theta_{e}=\frac{P}{2} \theta_{m}
\end{gathered}
$$

where $P$ is number of poles. While function $f\left(\theta_{e}\right)$ has form [10]:

$$
f\left(\theta_{e}\right)= \begin{cases}\frac{6}{\pi} \theta_{e}-1 & 0 \leq \theta_{e}<\frac{\pi}{3} \\ 1 & \frac{\pi}{3} \leq \theta_{e}<\pi \\ -\frac{6}{\pi} \theta_{e}+7 & \pi \leq \theta_{e}<\frac{4 \pi}{3} \\ -1 & \frac{4 \pi}{3} \leq \theta_{e}<2 \pi\end{cases}
$$


Applying Kirchoff's laws and algebraic transformations to equations (22)-(25) obtained:

$$
\begin{aligned}
& {\left[\begin{array}{c}
\dot{i}_{a} \\
\dot{i}_{b} \\
\dot{\omega}_{s} \\
\dot{\theta}_{m}
\end{array}\right]=\left[\begin{array}{cccc}
-\frac{R}{L} & 0 & 0 & 0 \\
0 & -\frac{R}{L} & 0 & 0 \\
0 & 0 & -\frac{B}{\mathbb{I}_{S}+\mathbb{I}_{P}} & 0 \\
0 & 0 & 1 & 0
\end{array}\right]\left[\begin{array}{c}
i_{a} \\
i_{b} \\
\omega_{s} \\
\theta_{m}
\end{array}\right]+} \\
& +\left[\begin{array}{ccc}
\frac{2}{3 L} & \frac{1}{3 L} & 0 \\
-\frac{1}{3 L} & \frac{1}{3 L} & 0 \\
0 & 0 & \frac{1}{\mathbb{I}_{S}+I_{P}} \\
0 & 0 & 0
\end{array}\right]\left[\begin{array}{c}
V_{a b}-E_{a b} \\
V_{b c}-E_{b c} \\
M_{e}
\end{array}\right]+ \\
& +\left[\begin{array}{c}
0 \\
0 \\
-\frac{M_{S}}{I_{S}+I_{P}} \\
0
\end{array}\right] \\
& {\left[\begin{array}{c}
i_{a} \\
i_{b} \\
i_{c} \\
\omega_{s} \\
\theta_{m}
\end{array}\right]=\left[\begin{array}{cccc}
1 & 0 & 0 & 0 \\
0 & 1 & 0 & 0 \\
-1 & -1 & 0 & 0 \\
0 & 0 & 1 & 0 \\
0 & 0 & 0 & 1
\end{array}\right]\left[\begin{array}{c}
i_{a} \\
i_{b} \\
\omega_{s} \\
\theta_{m}
\end{array}\right]}
\end{aligned}
$$

Based on equation (33) you can see that the inputs os system are voltages $V_{a b}$ i $V_{b c}$. They are coming from three phase inverter, which is shown on Figure 6. For the purpose of this work inverter can be simplified. Transistors was replaced witch switches. 


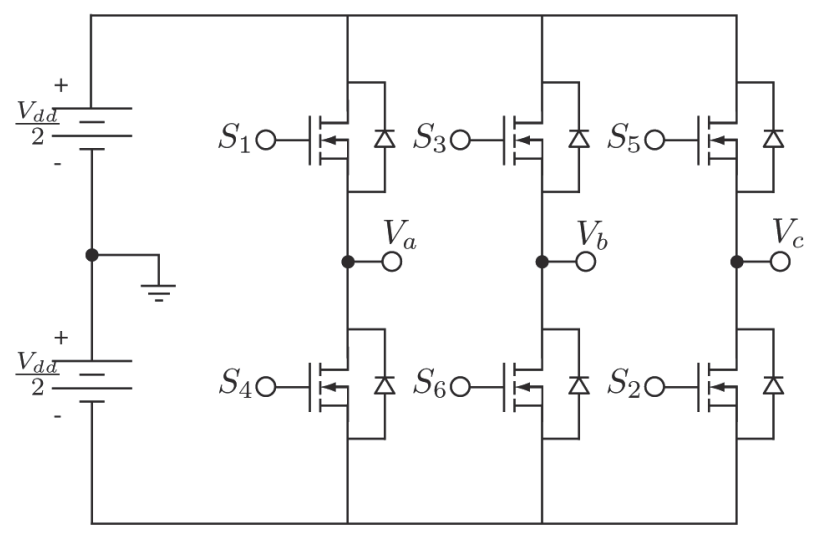

Fig. 6. Example of BLDC inverter

Therefore, following equations are obtained [12]:

$$
\begin{aligned}
& V_{a}=S_{1} \frac{V_{d d}}{2}-S_{4} \frac{V_{d d}}{2} \\
& V_{b}=S_{3} \frac{V_{d d}}{2}-S_{6} \frac{V_{d d}}{2} \\
& V_{c}=S_{5} \frac{V_{d d}}{2}-S_{2} \frac{V_{d d}}{2}
\end{aligned}
$$

where $V_{d d}$ is source voltage, and $S_{1}, S_{2}, \ldots, S_{6}$ are corresponding to state of switches in inverter. Switching table can be found in Table 1.

Table 1

Switching table

\begin{tabular}{|c|c|c|c|c|c|c|}
\hline$\theta_{e}$ & $S_{1}$ & $S_{2}$ & $S_{3}$ & $S_{4}$ & $S_{5}$ & $S_{6}$ \\
\hline $0^{\circ}$ & 0 & 0 & 0 & 0 & 1 & 1 \\
$60^{\circ}$ & 1 & 0 & 0 & 0 & 0 & 1 \\
$120^{\circ}$ & 1 & 1 & 0 & 0 & 0 & 0 \\
180 & 0 & 1 & 1 & 0 & 0 & 0 \\
$240^{\circ}$ & 0 & 0 & 1 & 1 & 0 & 0 \\
$300^{\circ}$ & 0 & 0 & 0 & 1 & 1 & 0 \\
\hline
\end{tabular}




\section{Li-poly battery}

Tricopter is powered by lithium-polymer battery. This is type of Li-ion cell, which is build from lithium alloys and conductive polymers. They are lightweight which makes them suitable for fling devices, and also characterised by a hight discharge current. Moreover they have grater energy density and have not „memory” effect, known from eg. Nickel-cadmium cells. Reason why model of battery should be include in overall model is dependency cell voltage from temperature, drawing current and state of charge. Change of battery potential has impact on angular speed of propellers which implicates difference in thrust and torque.

In scientific papers there are many elaborations about Li-poly cells, not only including chemical processes [5, 7], but also simplified models, which represents battery voltage from state of charge $[3,13,17,18]$. Author decided to choose simplified model without temperature compensations due to good cooling provided by airflow.

Model of battery is divided into two parts. First one is serving for approximation of state of charge. It is combined from capacitor and current source which represents respectively cell capacitance and current drawing of system. Scheme is shown on Figure 7. Effect of self-discharge was deliberately omitted because cells are discharging in rate about $8 \%$ monthly at $21^{\circ} \mathrm{C}[1]$.

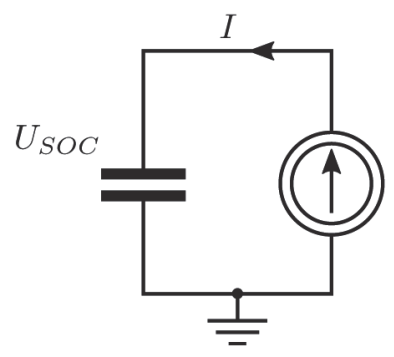

Fig. 7. Scheme of approximation of state of charge

Equation which represents state of charge is as follows:

$$
U_{S O C}=U_{p}-\frac{1}{C} \int I d t
$$

where:

C - is battery capacitance,

$I$ - is discharge current,

$U_{p}-$ is initial voltage in the range from 0 to 1 . 
The second part of model was developed based on Thévenin's theorem, which says, that any linear electrical network with voltage source and impedance can be replaced at terminals $\mathrm{AB}$ by an equivalent voltage source in series with matching impedance. In this case, voltage source is relative to $U_{S O C}$, and impedance has form of two resistors and capacitor. Scheme is shown on Figure 8.

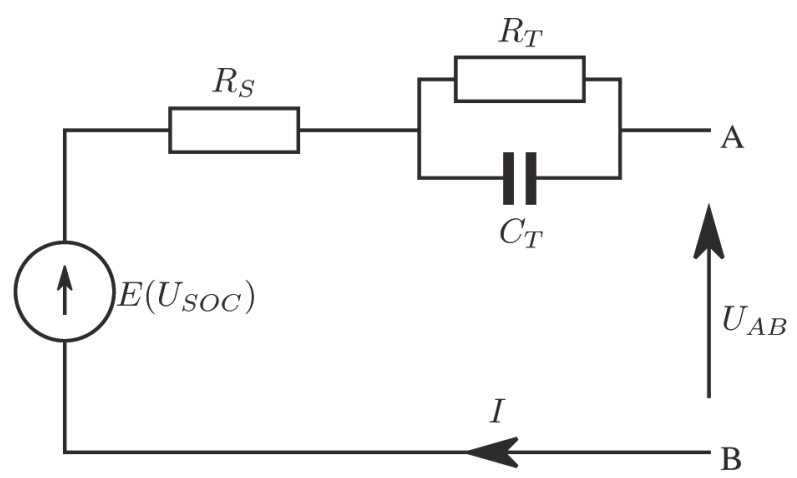

Fig. 8. Equivalent circuit of battery

According to Kirchoff's law:

$$
E\left(U_{S O C}\right)=I R_{S}+U_{R C}+U_{A B}
$$

where:

$$
\begin{aligned}
& I=\frac{U_{R C}}{R_{T}}+C_{T} \frac{d U_{R C}}{d t} \\
& \left\{\begin{array}{l}
\frac{d U_{R C}}{d t}=\frac{I}{C_{T}}-\frac{U_{R C}}{R_{T} C_{T}} \\
U_{R C}(0)=0
\end{array}\right. \\
& U_{R C}=I R_{T}-I R_{T} e^{-\frac{t}{R_{T} C_{T}}}
\end{aligned}
$$

after substitution:

$$
U_{A B}=E\left(U_{S O C}\right)-I R_{S}-I R_{T}+I R_{T} e^{-\frac{t}{R_{T} C_{T}}}
$$




\section{Flight dynamics of Tricopter}

Simplified models of Trirotor dynamics can be found in [14, 19, 21]. This representations absenting all important physical interactions, what is more, they are based on Euler angels, which may lead to gimbal lock effect or numerical errors. In this paper quaternions algebra is used to representation of angular movements, moreover additional force moments are included. What is more, standard aerospace frame - North East Down (NED) is used, in contrary to model in [21].

\subsection{Quaternions}

They are extension to imaginary numbers in 3D space. They were discovered by Irish mathematician - William Hamilton in 1843. On the beginning they were regarded as strange and unnatural, because their multiplication is noncommutative and they were discovered before matrices. They are used mainly to rotations in $\mathbb{R}^{3}$ space. Quaternions are represented in form:

$$
q=q_{0}+q_{1} i+q_{2} j+q_{3} k
$$

where: $q_{0}, q_{1}, q_{2}$ and $q_{3}$ are real numbers, symbols $i, j, k$ are quaternion units for which we defined:

$$
\begin{aligned}
& i^{2}=j^{2}=k^{2}=-1 \\
& i j=-j i=k, \quad j k=-k j=i, \quad k i=-i k=j
\end{aligned}
$$

Two Quaternions are equal only if they have exactly the same components. From equations (45) and (46) results that multiplication of quaternions in noncommutative.

Moreover quaternion conjugate is defined as:

$$
\bar{q}=q_{0}-q_{1} i-q_{2} j-q_{3} k
$$

and quaternion norm:

$$
N(q)=q_{0}^{2}+q_{1}^{2}+q_{2}^{2}+q_{3}^{2}
$$


Relationship between angular speed and quaternion is defined as [16]:

$$
\begin{aligned}
& {\left[\begin{array}{l}
\dot{q}_{0} \\
\dot{q}_{1} \\
\dot{q}_{2} \\
\dot{q}_{3}
\end{array}\right]=\frac{1}{2}\left[\begin{array}{cccc}
0 & -\omega_{p} & -\omega_{q} & -\omega_{r} \\
\omega_{p} & 0 & \omega_{r} & -\omega_{q} \\
\omega_{q} & -\omega_{r} & 0 & \omega_{p} \\
\omega_{r} & \omega_{q} & -\omega_{p} & 0
\end{array}\right]\left[\begin{array}{l}
q_{0} \\
q_{1} \\
q_{2} \\
q_{3}
\end{array}\right]+K_{q} \varepsilon\left[\begin{array}{c}
q_{0} \\
q_{1} \\
q_{2} \\
q_{3}
\end{array}\right]} \\
& \varepsilon=1-\left(q_{0}^{2}+q_{1}^{2}+q_{2}^{2}+q_{3}^{2}\right)
\end{aligned}
$$

where: $K_{q}$ is normalization gain, normally equal to 1 .

In the following part will be needed two more relationships. First between quaternion and rotation matrix and second between Euler angels and quaternion. They are respectively defined as:

$$
\begin{aligned}
& R=\left[\begin{array}{ccc}
q_{0}^{2}+q_{1}^{2}-q_{2}^{2}-q_{3}^{2} & 2\left(q_{1} q_{2}+q_{0} q_{3}\right) & 2\left(q_{1} q_{3}-q_{0} q_{2}\right) \\
2\left(q_{1} q_{2}-q_{0} q_{3}\right) & q_{0}^{2}-q_{1}^{2}+q_{2}^{2}-q_{3}^{2} & 2\left(q_{2} q_{3}+q_{0} q_{1}\right) \\
2\left(q_{1} q_{3}+q_{0} q_{2}\right) & 2\left(q_{2} q_{3}-q_{0} q_{1}\right) & q_{0}^{2}-q_{1}^{2}-q_{2}^{2}+q_{3}^{2}
\end{array}\right] \\
& {\left[\begin{array}{l}
\phi \\
\theta
\end{array}\right]=\left[\begin{array}{l}
\arctan \left(\frac{2\left(q_{2} q_{3}+q_{0} q_{1}\right)}{q_{0}^{2}-q_{1}^{2}-q_{2}^{2}+q_{3}^{2}}\right) \\
\arcsin \left(-2\left(q_{1} q_{3}-q_{0} q_{2}\right)\right) \\
\arctan \left(\frac{2\left(q_{1} q_{2}+q_{0} q_{3}\right)}{q_{0}^{2}+q_{1}^{2}-q_{2}^{2}-q_{3}^{2}}\right)
\end{array}\right]}
\end{aligned}
$$

Equations (51) and (52) are valid only for quaternions with unit norm.

\subsection{Tricopter's dynamics}

Tricopter flies due to thrust produce by three brushless motors with propellers. Two of them are spinning in clockwise direction, and the third in anticlockwise. It causes unbalance of aerodynamics torque and leads to autorotation of vehicle [21]. To eliminate this effect, one of rotors is placed on moving platform so tilted angle can be changed. 
Figure 9 shows forces generated by rotors $\left(T_{1}, T_{2}, T_{3}\right)$ and tilted angle $-\gamma$. Motors are placed in equal distances $(d)$ from mass centre, and angle between them is $120^{\circ}$.

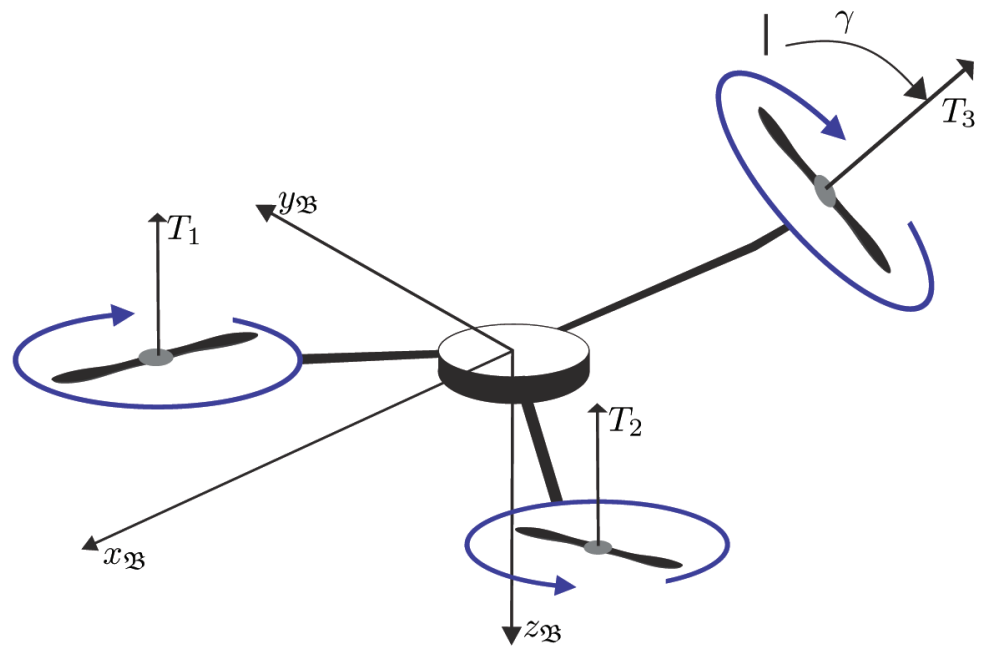

Fig. 9. Forces of Tricopter

Based on Figure 9 the resultant force and torque generated by $T_{i}$ for $i=1,2,3$ is:

$$
\begin{aligned}
\vec{F}^{\mathfrak{B}} & =\left[\begin{array}{c}
0 \\
-T_{3} \sin \gamma \\
-T_{1}-T_{2}-T_{3} \cos \gamma
\end{array}\right] \\
\vec{M}_{T}^{\mathfrak{B}} & =\left[\begin{array}{c}
\left(T_{2}-T_{1}\right) d \sin \frac{\pi}{3} \\
\left(T_{1}+T_{2}\right) d \cos \frac{\pi}{3}-T_{3} d \cos \gamma \\
T_{3} d \sin \gamma
\end{array}\right]
\end{aligned}
$$

according to (7) $T_{i}=C_{T} \rho n_{s_{i}}^{2} D^{4}$, substituting $n_{s_{i}}=\frac{\omega_{s_{i}}}{2 \pi}$ for $i=1,2,3$ obtained:

$$
T_{i}=C_{T} \rho \frac{\omega_{s_{i}}^{2}}{4 \pi^{2}} D^{4}
$$


which after alternate into (53) and (54) gives:

$$
\begin{gathered}
\vec{F}^{\mathfrak{B}}=-C_{T} \rho \frac{D^{4}}{4 \pi^{2}}\left[\begin{array}{c}
0 \\
\omega_{s_{3}}^{2} \sin \gamma \\
\omega_{s_{1}}^{2}+\omega_{s_{2}}^{2}+\omega_{s_{3}}^{2} \cos \gamma
\end{array}\right] \\
\vec{M}_{T}^{\mathfrak{B}}=C_{T} \rho d \frac{D^{4}}{4 \pi^{2}}\left[\begin{array}{c}
\frac{\sqrt{3}}{2}\left(\omega_{s_{2}}^{2}-\omega_{s_{1}}^{2}\right) \\
\frac{1}{2}\left(\omega_{s_{1}}^{2}+\omega_{s_{2}}^{2}\right)-\omega_{s_{3}}^{2} \cos \gamma \\
\omega_{s_{3}}^{2} \sin \gamma
\end{array}\right]
\end{gathered}
$$

Torque vector (57) is not only one moment of force in this system. Additional moments are created by moving tilted, tail, motor. This moment is dependent on angular acceleration and inertia of tail engine $[14,21]$. It produces rotation in $x_{\mathfrak{B}}$ axis and is expressed as follows:

$$
\vec{M}_{\gamma}^{\mathfrak{B}}=\left[-I_{z n} \ddot{\gamma}, 0,0\right]^{T}
$$

During rotation of tail rotor occurs gyroscopic effect. This torque depends on propeller momentum $[9,14,21]$ :

$$
\vec{M}_{z}^{\mathfrak{B}}=I_{s} \omega_{s_{3}} \dot{\gamma}[0, \sin \gamma,-\cos \gamma]^{T}
$$

Moreover, in this system, is presented another torque which is created by change of momentum, which is result of difference of propeller angular speed.

$$
\vec{M}_{\omega}^{\mathfrak{B}}=I_{s}\left[0,-\dot{\omega}_{s_{3}} \sin \gamma, \dot{\omega}_{s_{2}}-\dot{\omega}_{s_{1}}-\dot{\omega}_{s_{3}} \cos \gamma\right]^{T}
$$

What is more, a propeller shafts encounters aerodynamics drag, which based on third Newton law generates torque in aerodynamic centre [21]. The result is rotation in 
counter direction then propeller. Detailed calculations can be found in chapter 2. The outcome is equation (21), and vector is defined as:

$$
\vec{M}_{P}^{\mathfrak{B}}=c_{M_{P}} \rho d \frac{D^{4}}{4 \pi^{2}}\left[0, \omega_{s_{3}}^{2} \sin \gamma, \omega_{s_{2}}^{2}-\omega_{s_{1}}^{2}-\omega_{s_{3}}^{2} \cos \gamma\right]^{T}
$$

The result of adding equations from (57) to (61) is resultant torque:

$$
\vec{M}^{\mathfrak{B}}=\left[\begin{array}{c}
\frac{C_{1} \sqrt{3}}{2}\left(\omega_{s_{2}}^{2}-\omega_{s_{1}}^{2}\right)-I_{z n} \ddot{\gamma} \\
\frac{C_{1}}{2}\left(\omega_{s_{1}}^{2}+\omega_{s_{2}}^{2}\right)-C_{1} \omega_{s_{3}}^{2} c_{\gamma}+I_{s} \omega_{s_{3}} \dot{\gamma} s_{\gamma}-I_{s} \dot{\omega}_{s_{3}} s_{\gamma}+C_{2} \omega_{s_{3}}^{2} s_{\gamma} \\
C_{1} \omega_{s_{3}}^{2} s_{\gamma}-I_{s} \omega_{s_{3}} \dot{\gamma} c_{\gamma}+I_{s}\left(\dot{\omega}_{s_{2}}-\dot{\omega}_{s_{1}}-\dot{\omega}_{s_{3}} c_{\gamma}\right)+C_{2}\left(\omega_{s_{2}}^{2}-\omega_{s_{1}}^{2}-\omega_{s_{3}}^{2} c_{\gamma}\right)
\end{array}\right]
$$

where $C_{1}=C_{T} \rho d \frac{D^{4}}{4 \pi^{2}}, C_{2}=C_{M_{P}} \rho d \frac{D^{4}}{4 \pi^{2}}, s_{\gamma}, c_{\gamma}$ is $\sin \gamma$ and $\cos \gamma$.

Six degree of freedom equations of motions are defined as [6]:

$$
\begin{aligned}
& \vec{F}^{\mathfrak{B}}=\dot{m} V_{b}+m \dot{V}_{b}+\omega \times\left(m V_{b}\right)-R^{T}[0,0, m g]^{T} \\
& \vec{M}^{\mathfrak{B}}=\dot{I} \omega+\amalg \dot{\omega}+\omega \times(\amalg \omega)
\end{aligned}
$$

where $m$ is Tricopter's mass, $V_{b}$ is his speed in body frame, $\omega=\left[\omega_{p}, \omega_{q}, \omega_{r}\right]^{T}$ is angular speed and II is his inertia. Because Tricopter's mass is constant during flight, and his construction is stiff so his inertia is also constant. Above equations are transformed into:

$$
\begin{aligned}
& \dot{V}_{b}=\frac{1}{m} \vec{F}^{\mathfrak{B}}-\omega \times V_{b}+R^{T}[0,0, g]^{T} \\
& \dot{\omega}=\mathbb{I}^{-1}\left(\vec{M}^{\mathfrak{B}}-\omega \times(\mathbb{I} \omega)\right)
\end{aligned}
$$

More interesting is speed in Earth frame then body axes. Relationship between them is following:

$$
\dot{V}=R \dot{V}_{b}
$$


This differential equations represents mathematical model of Tricopter's dynamics:

$$
\left\{\begin{aligned}
& \dot{S}=V \\
& \dot{V}=R \dot{V}_{b} \\
& \dot{V}_{b}=\frac{1}{m} \vec{F}^{\mathfrak{B}}-\omega \times V_{b}+R^{T}[0,0, g]^{T} \\
& \dot{\omega}=\mathbb{I}^{-1}\left(\vec{M}^{\mathfrak{B}}-\omega \times(I \omega)\right) \\
& {\left[\begin{array}{c}
\dot{q}_{0} \\
\dot{q}_{1} \\
\dot{q}_{2} \\
\dot{q}_{3}
\end{array}\right]=\frac{1}{2}\left[\begin{array}{cccc}
0 & -\omega_{p} & -\omega_{q} & -\omega_{r} \\
\omega_{p} & 0 & \omega_{r} & -\omega_{q} \\
\omega_{q} & -\omega_{r} & 0 & \omega_{p} \\
\omega_{r} & \omega_{q} & -\omega_{p} & 0
\end{array}\right]\left[\begin{array}{l}
q_{0} \\
q_{1} \\
q_{2} \\
q_{3}
\end{array}\right]+K_{q} \varepsilon\left[\begin{array}{l}
q_{0} \\
q_{1} \\
q_{2} \\
q_{3}
\end{array}\right] }
\end{aligned}\right.
$$

where $S$ is location in Earth frame.

\section{Summary}

This paper presents six degrees of freedom analytical model of Tricopter's dynamics. It is based on quaternion's algebra instead Euler's angles, this leads to eliminations of gimbal-lock effect in numeric simulation. Moreover, to describe full dynamics, propulsion and electricity subsystems are demonstrated. Next step will be models validation and then synthesis of controller.

\section{References}

[1] Abe H., Murai T., Zaghib K., Vapor-grown carbon fiber anode for cylindrical lithium ion rechargeable batteries, Journal of Power Sources, 1999, Vol. 77, No. 2, pp. 110-115, DOI: 10.1016/S0378-7753(98)00158-X.

[2] Bose T., Airbreathing Propulsion: An Introduction, Springer, 2012, DOI: 10.1007/978-14614-3532-7.

[3] Chen M., Rincon-Mora G., Accurate electrical battery model capable of predicting runtime and I-V performance", Energy Conversion, IEEE Transactions on 21.2 (June 2006), pp. 504-511, DOI: 10.1109/TEC.2006.874229.

[4] De S. et al., Low inductance axial flux BLDC motor drive for more electric aircraft, in: Aerospace Conference, 2011 IEEE. Mar. 2011, pp. 1-11, DOI: 10.1109/AERO.2011.5747464.

[5] Doyle M., Newman J., The use of mathematical modeling in the design of lithium/polymer battery systems, in: Electrochimica Acta, 1995, 40.13-14, International symposium on polymer electrolytes, pp. 2191-2196, DOI: 10.1016/0013-4686(95)00162-8. 
[6] Dreier M.E., Introduction to Helicopter and Tiltrotor Simulation, American Institute of Aeronautics and Astronautics, 2007.

[7] Gomadam P.M. et al., Mathematical modeling of lithium-ion and nickel battery systems, Journal of Power Sources, 2002, Vol. 110, No. 2, pp. 267-284, DOI: 10.1016/S03787753(02)00190-8.

[8] Jeon Y. et al., A new simulation model of BLDC motor with real back EMF waveform, in: Computers in Power Electronics, 2000. COMPEL 2000. The 7th Workshop on. 2000, pp. 217-220, DOI: 10.1109/CIPE.2000.904719.

[9] Kendoul F., Fantoni I., Lozano R., Modeling and Control of a Small Autonomous Aircraft Having Two Tilting Rotors, in: Robotics, IEEE Transactions on 22.6, December 2006, pp. 1297-1302, DOI: 10.1109/TRO.2006.882956.

[10] Martinez-Alvarado R. et al., Modeling and simulation of a propeller-engine system for Unmanned Aerial Vehicles, in: Power, Electronics and Computing (ROPEC), 2013 IEEE International Autumn Meeting on. Nov. 2013, pp. 1-6, DOI: 10.1109/ROPEC.2013.6702722.

[11] Phillips W.F., Mechanics of Flight, John Wiley \& Sons, 2009.

[12] Prasad G. et al., Modelling and Simulation Analysis of the Brushless DC Motor by using $M A T L A B$, International Journal of Innovative Technology and Exploring Engineering, Oct. 2012, 15.

[13] Rahimi-Eichi H., Baronti F., Chow M.-Y., Modeling and online parameter identification of Li-Polymer battery cells for SOC estimation, in: Industrial Electronics (ISIE), 2012 IEEE International Symposium on. May 2012, pp. 1336-1341, DOI: 10.1109/ISIE.2012.6237284.

[14] Salazar-Cruz S. et al., Real-Time Control of a Small-Scale Helicopter Having Three Rotors, in: Intelligent Robots and Systems, 2006 IEEE/RSJ International Conference on October 2006, pp. 2924-2929, DOI: 10.1109/IROS.2006.282145.

[15] Singh Patel V., Pandey A., Modeling and Performance Analysis of PID Controlled BLDC Motor and Different Schemes of PWM Controlled BLDC Motor, in: International Journal of Scientific and Research Publications, Apr. 2013.

[16] The MathWorks Inc. 6DOF (Quaternion). 2015, http://www.mathworks.com/help/aeroblks/ 6dofquaternion.html (visited on 04/08/2015).

[17] The MathWorks Inc. Battery. 2015. URL: http://www.mathworks.com/help/physmod/sps/ powersys/ref/battery.html (visited on 04/08/2015).

[18] Yao L.W. et al., Modeling of lithium-ion battery using MATLAB/simulink, in: Industrial Electronics Society, IECON 2013 - 39th Annual Conference of the IEEE. Nov. 2013, pp. 1729-1734, DOI: 10.1109/IECON.2013.6699393.

[19] Yoo D.-W. et al., Dynamic modeling and control system design for Tri-Rotor UAV, in: Systems and Control in Aeronautics and Astronautics (ISSCAA), 2010 3rd International Symposium on. June 2010, pp. 762-767, DOI: 10.1109/ISSCAA.2010.5632868.

[20] Źrebiec J., Modelling and control of Tricopter - an Unmanned Aerial Vehicle, Master of science thesis, AGH, Kraków 2015.

[21] Źrebiec J., Włodarz R., Laboratory model of Tricopter, Bachelors thesis, AGH, Kraków 2013, p. 16. 